Proceedings

\title{
Infrared Non-Destructive Testing via Semi-Nonnegative Matrix Factorization ${ }^{+}$
}

\author{
Bardia Yousefi *, Clemente Ibarra-Castanedo and Xavier P.V. Maldague \\ Computer vision and systems laboratory, Department of Electrical and Computer Engineering, \\ Laval University, Quebec City G1V 0A6, QC, Canada; IbarraC@gel.ulaval.ca (C.I.-C.); \\ Xavier.Maldague@gel.ulaval.ca (X.P.V.M.) \\ * Correspondence: Bardia.Yousefi.1@ulaval.ca \\ + Presented at the 15th International Workshop on Advanced Infrared Technology and Applications \\ (AITA 2019), Florence, Italy, 17-19 September 2019.
}

Published: 20 September 2019

\begin{abstract}
Detection of subsurface defects is undeniably a growing subfield of infrared nondestructive testing (IR-NDT). There are many algorithms used for this purpose, where non-negative matrix factorization (NMF) is considered to be an interesting alternative to principal component analysis (PCA) by having no negative basis in matrix decomposition. Here, an application of Semi non-negative matrix factorization (Semi-NMF) in IR-NDT is presented to determine the subsurface defects of an Aluminum plate specimen through active thermographic method. To benchmark, the defect detection accuracy and computational load of the Semi-NMF approach is compared to stateof-the-art thermography processing approaches such as: principal component thermography (PCT), Candid Covariance-Free Incremental Principal Component Thermography (CCIPCT), Sparse PCT, Sparse NMF and standard NMF with gradient descend (GD) and non-negative least square (NNLS). The results show $86 \%$ accuracy for 27.5 s computational time for SemiNMF, which conclusively indicate the promising performance of the approach in the field of IR-NDT.
\end{abstract}

Keywords: subsurface defect detection; Semi Non-negative matrix factorization (Semi-NMF); infrared non-destructive testing (IR-NDT)

\section{Introduction}

A matrix decomposition processed by Non-negative matrix factorization (NMF) leads to decomposing an input matrix into two low-rank non-negative matrices similar to principal component analysis (PCA) but with non-negative matrix constraints [1]. The NMF shows a similar behavior as a clustering algorithm and a known reason to be a subfield of data-mining and pattern recognition [1,2]. Matrix factorization has been used for infrared non-destructive testing (IR-NDT) and compared to PCA and archetypal analysis (AA) to assess its advantages and pitfalls as reported in [5]. This analysis continued in [6] where NMF was applied to cultural heritage objects and buildings using gradient descend (GD) and non-negative least square (NNLS) and demonstrating the good performance of such algorithms for detecting subsurface defects. Here, we present Semi-NMF using NNLS and based on gradient descent rule (Ruls) methods to detect subsurface defects in Aluminum. 


\section{Materials and Methods}

\subsection{Testing Specimen and Experimental Setup}

Aluminum is extensively employed in many industrial and aerospace structures, it is also considered to be a benchmark for the performance evaluation of thermographic methods under laboratory conditions. The data-set was assembled using following an experimental scenario regarding and acquisition parameters as described in [7]. The inspection was conducted from the front side of the specimen in reflection mode (having a depth range from 0.2 to $1 \mathrm{~mm}$ ). Two photographic flashes were used (Balcar FX 60, $5 \mathrm{~ms}$ thermal pulse, $6.4 \mathrm{~kJ} / \mathrm{flash}$ ) and a MWIR infrared camera (Santa Barbara Focal plane (nitrogen cooled, InSb, $320 \times 256$ pixels). The acquisition parameters were tuned as: Sampling rate, $\boldsymbol{f}_{\boldsymbol{s}}=157 \mathrm{~Hz}$; Duration, $\boldsymbol{t}_{\boldsymbol{a c q}}=6.37 \mathrm{~s}$; Time step, $\boldsymbol{D}_{\boldsymbol{t}}=0.025$ $\mathrm{s}$; truncation window, $w_{s}(t)=6.37 \mathrm{~s}$; total number of frames $=250$ (Figure 1a). Figure $1 \mathrm{~b}$ shows the scheme of the specimen which has been utilized [7].

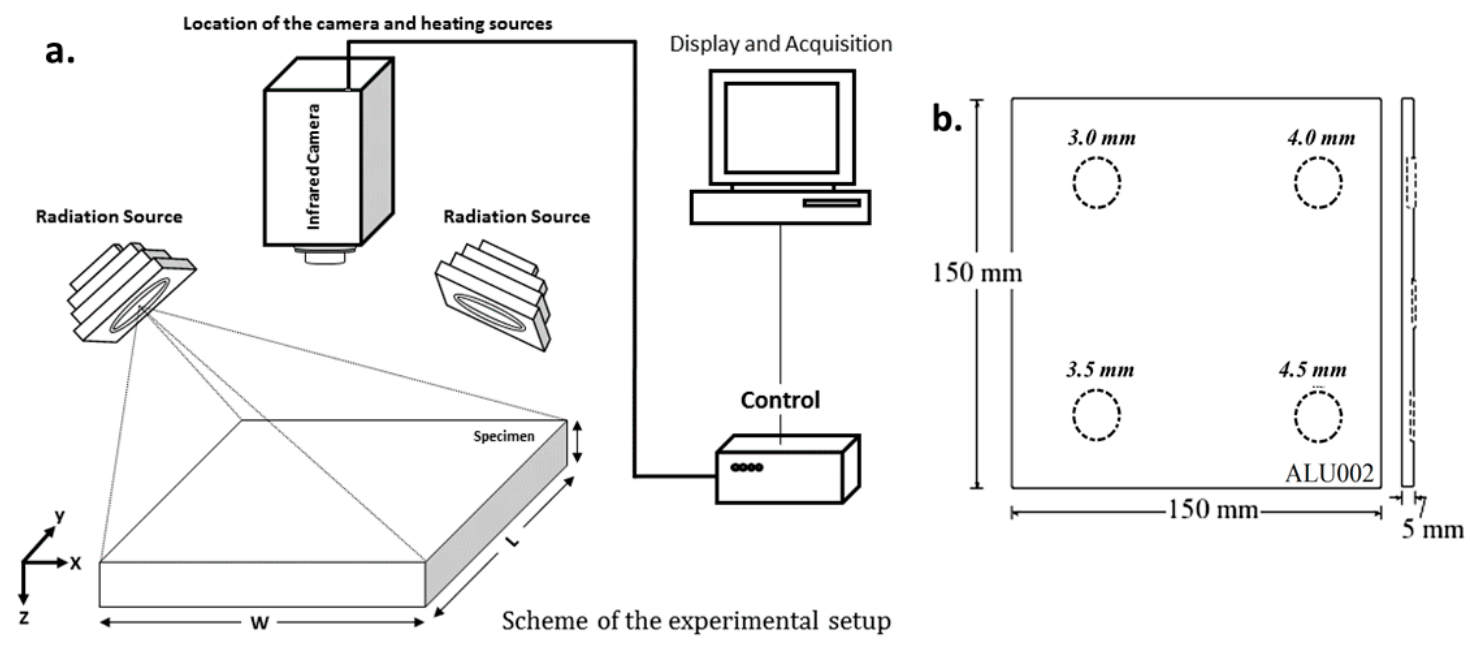

Figure 1. Experimental setup and schematic of Aluminum plate are presented.

\subsection{Methodology}

This approach shows a presentation of factorization in IR-NDT. Factorization considers to be shown by following formula:

$$
X \approx \mathbf{B H}^{T},
$$

Where the input matrix $\boldsymbol{X}=\left(\boldsymbol{x}_{1}, \ldots, \boldsymbol{x}_{\boldsymbol{n}}\right)$ shows a collection of $\boldsymbol{n}$ data vectors in its columns correspond to $\boldsymbol{n}$ vectorized infrared images, $\boldsymbol{X} \in \boldsymbol{R}^{\boldsymbol{p} \times \boldsymbol{n}}$, and $\mathbf{H} \in \boldsymbol{R}^{\boldsymbol{n} \times \boldsymbol{k}}$. This represents the singular value decomposition (SVD), where there are no limitations on the signs of $\boldsymbol{B}$ and $\boldsymbol{H}$, also non constrain for input matrix $\boldsymbol{X}$. NMF assumes that matrices $\boldsymbol{X}, \boldsymbol{B}$, and $\boldsymbol{H}$ are not negative, but when the data matrix is unconstrained (i.e., when mixed signs exist among the matrices) NMF is referred to be called Semi-NMF, which $\boldsymbol{H}$ is restricted to be non-negative but there is no restriction for matrix $\boldsymbol{B}$ [11]. This provides freedom to basis matrix (similar to PCT) which is restricted by a coefficient matrix. The NMF directly associates with clustering [1,3], Semi-NMF can be modified from this perspective, where if Semi-NMF performs grouping (similar to K-means clustering) on input infrared data of $\boldsymbol{X}$, the $\boldsymbol{B}$ and $\boldsymbol{H}$ can be represented by a cluster centroids matrix $\boldsymbol{B}=\left(\boldsymbol{\beta}_{1}, \boldsymbol{\beta}_{2}, \ldots, \boldsymbol{\beta}_{\boldsymbol{k}}\right)$, and by cluster indicators; $\boldsymbol{\eta}_{\boldsymbol{i} \boldsymbol{k}}=\mathbf{1}$ if $\boldsymbol{x}_{\boldsymbol{i}}$ belonging to cluster $\boldsymbol{c}_{\boldsymbol{k}} ; \boldsymbol{\eta}_{\boldsymbol{i} \boldsymbol{k}}=\mathbf{0}$ otherwise. This can be shown similar to a clustering objective function as:

$$
J_{k-m e a n s}=\sum_{i=1}^{n} \sum_{k=1}^{K} \eta_{i k}\left\|x_{i}-\beta_{k}\right\|^{2}=\left\|X-B H^{T}\right\|^{2},
$$


Let $\|$.$\| denotes the \boldsymbol{\ell}_{2}$-norm of a vector and $\|$.$\| represents Frobenius norm of a matrix. This$ indicates that K-means clustering objective can be alternatively viewed as an objective function for matrix approximation, which indicates the clustering among subsurface defects and other regions in the input matrix. In the case of relaxation, following optimization allows $\boldsymbol{\eta}_{i j}$ to range over values in $(0,1)$, or values in $(\mathbf{0}, \infty)$, which yields the Semi-NMF type of matrix factorization.

\section{Results and Conclusions}

The presented thermography analysis has been performed in a PC (Intel(R) Core(TM) i5 CPU, 3.20GHz, RAM 16.00GB, 64 bit Operating System) and using MATLAB computer programming. Figure 2 presents selected results of subsurface defect detection using semi-NMF computed by two computational methods: non-negative least square (SemiNMF-nnls, Figure 2h), and gradient descent rules (SemiNMF-Ruls, Figure 2i) and compared to the state-of-the-art approaches such as NMF (Figure 2a), PCT [8] (Figure 2b), NMF-gd (Figure 2c), NMF-nnls [6] (Figure 2d), Sparse-PCT [10] (Figure 2e), Candid Covariance-Free Incremental Principal Component Thermography (CCIPCT) [9] (Figure 2g), and Sparse-NMF (Figure 2e) [12]. We selected $k=10$ for most of this analyses corresponding to an $80 \%$ variance via decomposition method for all methods. The qualitative results of SemiNMF-nnls indicated considerable accuracy relative to state-of-the-art techniques and SemiNMF-Ruls [11]. To benchmark, we used a binary image as ground truth (GT) and a reference for our calculation. A metric called Intersection over Union (IoU), also denoted as Jaccard index, is employed to quantify the percent overlap between the GT and our prediction output and measured by the number of common pixels between the GT and resulting masks divided by overall number of pixels. The quantitative results in Table 1 indicate an $86 \%$ IoU accuracy with $27.5 \mathrm{~s}$ of computational load for SemiNMF-nnls compared to the state-of-the-art thermographic approaches. For instance, NMF and PCT have IoU range of 95\% (0.25 s) and 91\% (0.56 s), respectively. NMF-gd and NMF-nnls were likewise relatively accurate having $87 \%$ (14.81 s) and 83\% (45.18 s), respectively.

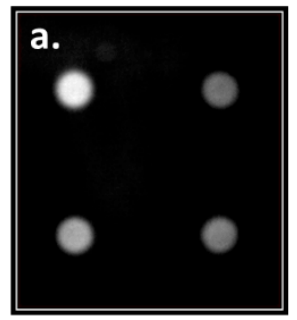

NMF

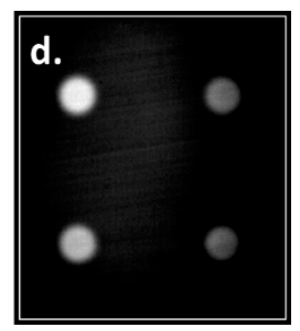

NMF-nnls

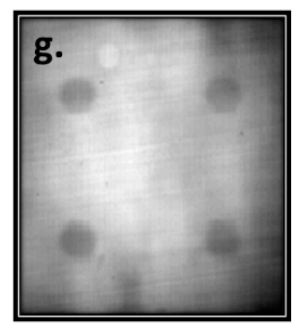

CCIPCT

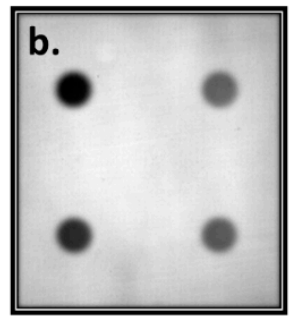

PCT

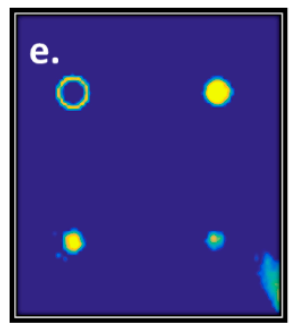

Sparse-PCT

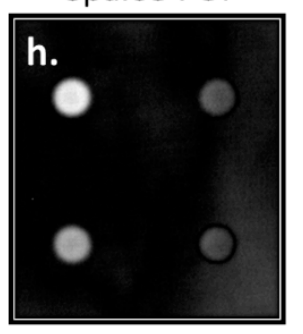

Semi-NMF-nnls

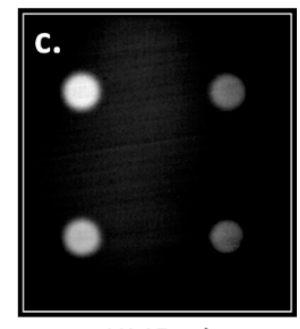

NMF-gd

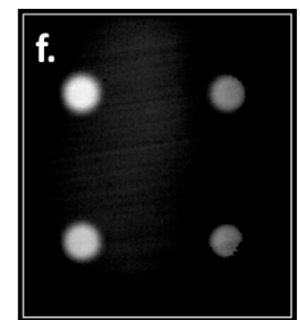

Sparse-NMF

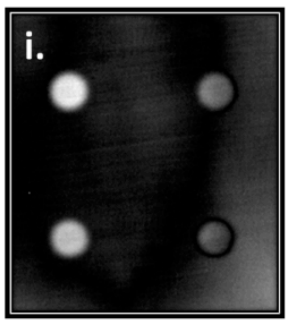

Semi-NMF-Ruls

Figure 2. Evaluation of the qualitative accuracy of Semi-NMF (h.,i.) versus the state-of-the-art approaches (a.-g.) in thermography. 
Table 1. The table shows the IoU metric and computational load of each algorithm.

\begin{tabular}{ccc}
\hline Method & Computational Load (s) & IoU (\%) \\
\hline CCIPCT & 0.04 & 0.2 \\
PCT & 0.25 & 0.91 \\
NMF & 0.56 & 0.95 \\
Standard-NMF-gd & 14.81 & 0.87 \\
Standard-NMF-nnls & 45.18 & 0.83 \\
Sparse-PCT & 18.31 & 0.4 \\
Sparse-NMF & 39.52 & 0.93 \\
SemiNMF-Ruls & 27.56 & 0.53 \\
SemiNMF-nnls & 27.5 & 0.86 \\
\hline
\end{tabular}

The accuracy of SemiNMF, Sparse NMF, and Sparse PCT might be lesser than NMF and PCT which is due to non-noisy condition of IR-NDT (no additive noise), while sparse methods have better resistance against additive noise. As future work, further analysis of these methods will be conducted to determine their robustness in the presence of noise.

Funding: This research was conducted under tier-1 Canadian Research Chair in Multipolar Infrared Vision (MIVIM) at Computer Vision and Systems Laboratory (CVSL), Laval University.

Conflicts of Interest: The authors declare no conflict of interest.

\section{References}

1. Lee, D.D.; Seung, H.S. Learning the parts of objects by non-negative matrix factorization. Nature 1999, 401, 788-791.

2. Berman, A.; Plemmons, R.J. Inverses of nonnegative matrices. Linear Multilinear Algebra 1974, 2, 161-172.

3. Lee, D.D.; Seung, H.S. Algorithms for non-negative matrix factorization. Adv. Neural Inf. Process. Syst. 2001, 401, 556-562.

4. Paatero, P.; Tapper, U. Positive matrix factorization: A non-negative factor model with optimal utilization of error estimates of data values. Environmetrics 1994, 5, 111-126.

5. Marinetti, S.; Finesso, L.; Marsilio, E. Matrix factorization methods: Application to thermal NDT/E. NDT E Int. 2006, 39, 611-616.

6. Yousefi, B.; Sfarra, S., Ibarra-Castanedo, C.; Avdelidis, N.P.; Maldague, X.P. Thermography data fusion and nonnegative matrix factorization for the evaluation of cultural heritage objects and buildings. J. Therm. Anal. Calorim. 2019, 136, 943-955.

7. Ibarra-Castanedo, C.; Maldague, X.P. Defect depth retrieval from pulsed phase thermographic data on Plexiglas and aluminum samples. In Thermosense XXVI; International Society for Optics and Photonics: Bellingham, WA, USA, 2004; Volume 5405, pp. 348-356.

8. Rajic, N. Principal component thermography for flaw contrast enhancement and flaw depth characterisation in composite structures. Compos. Struct. 2002, 58, 521-528.

9. Yousefi, B.; Sfarra, S.; Ibarra-Castanedo, C.; Maldague, X.P. Comparative analysis on thermal nondestructive testing imagery applying Candid Covariance-Free Incremental Principal Component Thermography (CCIPCT). Infrared Phys. Technol. 2017, 85, 163-169.

10. Yousefi, B.; Sfarra, S.; Sarasini, F.; Ibarra-Castanedo, C.; Maldague, X.P. Low-rank sparse principal component thermography (sparse-PCT): Comparative assessment on detection of subsurface defects. Infrared Phys. Technol. 2019, 98, 278-284.

11. Ding, C.; Li, T.; Jordan, M. Convex and Semi-Nonnegative Matrix Factorizations. IEEE Trans. Pattern Anal. Mach. Intell. 2008, 32, 45-55. 
12. Yousefi, B.; Ibarra-Castanedo, C.; Maldague, X.P. Application of Sparse Non-Negative Matrix Factorization in infrared non-destructive testing. In Proceedings of the 3rd Asian Conference on Quantitative Infrared Thermography, Tokyo, Japan, 1-5 July 2019. Available online: https://qirtasia2019.com/wpcontent/uploads/2019/05/Program20190511.pdf (accessed on 20 September 2019).

(C) 2019 by the authors. Licensee MDPI, Basel, Switzerland. This article is an open access article distributed under the terms and conditions of the Creative Commons Attribution (CC BY) license (http://creativecommons.org/licenses/by/4.0/). 